\title{
Scattering from Two-Dimensional Objects of Varying Shape Combining the Multilevel Fast Multipole Method (MLFMM) with the Stochastic Galerkin Method (SGM)
}

\author{
Zdravko Zubac ${ }^{1}$, Daniel De Zutter ${ }^{1}$, Fellow IEEE, Dries Vande Ginste ${ }^{1}$, Senior Member, IEEE
}

\begin{abstract}
In this letter, the Multilevel Fast Multipole Method (MLFMM) is combined with the Polynomial Chaos Expansion (PCE) approach to model the stochastic variations of a scatterer. In particular, it is demonstrated how the Stochastic Galerkin Method (SGM) can be combined with an MLFMM accelerated Method of Moments (MoM) and how the beneficial effects of the MLFMM for electromagnetically large scatterers are retained in the stochastic case.
\end{abstract}

Index Terms-Stochastic Galerkin Method (SGM), Multilevel Fast Multipole Method (MLFMM), scattering, Method of Moments (MoM)

\section{INTRODUCTION}

Electromagnetic scattering analysis relies on exact data of the considered structures such as material parameters and geometry. In reality, these data exhibit variability and modeling of these uncertainties can be achieved by combining electromagnetic analysis and stochastic analysis methods. The standard approach is to assume that the parameters of interest are random variables with a predefined probability density function (PDF). The goal is to determine the statistics of output parameters of interest, such as the bistatic radar cross-section (RCS) and the current distribution on the scatterer.

A standard way to analyze the effect of variability is by means of straightforward Monte Carlo (MC) simulations which, unfortunately, show quite slow convergence at a rate $1 / \sqrt{N_{s}}$, where $N_{s}$ is the number of separate runs of the code. As an alternative, spectral methods based on polynomial chaos expansions (PCE) using orthogonal polynomials depending on the particular distribution of the random variables were proposed [1]. Roughly speaking, these methods come in two flavors: the non-intrusive ones, such as the Stochastic Collocation Method (SCM) [2] and the intrusive methods, such as the Stochastic Galerkin method (SGM) [3]. Whereas nonintrusive methods rely on a standard deterministic solver to obtain the statistical information, intrusive methods require the development of a dedicated new solver. PCE-based methods are already used for variability analysis of (on-chip) interconnects [4] [5]. Superiority of PCE-based methods over MC simulations for scattering problems is, e.g., demonstrated in [6] and will not be repeated in this letter.

\footnotetext{
${ }^{1}$ Electromagnetics Group, Department of Information Technology, Ghent University/iMinds, Sint-Pietersnieuwstraat 41, 9000 B-Gent, Belgium. E-mail: zdravko.zubac@UGent.be, Tel.: +32 92643323
}

In this letter, we will use the SGM approach to model stochastic scattering problem by means of an integral equation. As indicated in [5], applying SGM to integral equations combined with the Method of Moments (MoM), leads to solving a large deterministic problem. In order to decrease the computational complexity, the Multilevel Fast Multipole Method (MLFMM) [7] is invoked. To the best knowledge of the authors, this letter is the first to discuss the combination of MLFMM with the intrusive SGM for the stochastic analysis of scattering problems. To assess the benefits of the MLFMM approach, we compare it to the standard MoM approach combined with SGM. Section II discusses the theoretical framework, combining the Electric Field Integral Equation (EFIE), its MoM solution, the SGM with its expansion of all variables in the proper stochastic basis functions and the way the MLFMM can be adapted to the stochastic approach. The numerical example in Section III illustrates the acceleration obtained by introducing the MLFMM in the stochastic approach.

\section{THE STOCHASTIC SCATTERING PROBLEM}

We consider two-dimensional frequency domain scattering by perfect electrically conducting (PEC) objects, residing in free space, with a stochastically defined geometry. This geometric variability of the scatterer is described by a set of $M$ independent random variables which are collected in the vector $\boldsymbol{\xi}=\left[\begin{array}{llll}\xi_{1} & \xi_{2} & \ldots & \xi_{M}\end{array}\right]$ with domain $\Omega$. The $z$-axis is the axis of invariance. The incident wave is a TM-polarized plane wave with electric field $\boldsymbol{E}^{i}=E^{i} \mathbf{u}_{z}$. To determine the scattered field, we apply an electric field integral equation (EFIE) solved by the MoM. To this end, the scatterer is divided into $N$ segments and pulse basis functions for the current distribution are used. As a result, a stochastic linear system equation is obtained:

$$
\bar{Z}(\boldsymbol{\xi}) \boldsymbol{I}(\boldsymbol{\xi})=\boldsymbol{V}(\boldsymbol{\xi})
$$

Next, all elements of (1) are represented via a PCE, from which we get:

$$
\sum_{k=0}^{K} \boldsymbol{V}_{k} \phi_{k}(\boldsymbol{\xi})=\sum_{k=0}^{K} \sum_{l=0}^{K} \overline{\boldsymbol{Z}}_{k} \boldsymbol{I}_{l} \phi_{k}(\boldsymbol{\xi}) \phi_{l}(\boldsymbol{\xi}),
$$

where $\bar{Z}_{k}, \boldsymbol{V}_{k}$ and $\boldsymbol{I}_{l}$ represent the expansion coefficients of system matrix $\overline{\boldsymbol{Z}}$, RHS $\boldsymbol{V}$ and unknown current density $\boldsymbol{I}$, resp. $\phi_{k}(\boldsymbol{\xi})$ and $\phi_{l}(\boldsymbol{\xi})$ are multivariate polynomials that are orthonormal with respect to the PDF $W(\boldsymbol{\xi})$ of the random 
vector $\boldsymbol{\xi}$. The expansion coefficients of any quantity $\boldsymbol{f}(\boldsymbol{\xi})$ are obtained via:

$$
\boldsymbol{f}_{k}=<\boldsymbol{f}(\boldsymbol{\xi}), \phi_{k}(\boldsymbol{\xi})>,
$$

where $<f(\boldsymbol{\xi}), g(\boldsymbol{\xi})>$ represents an inner product defined as:

$$
<f(\boldsymbol{\xi}), g(\boldsymbol{\xi})>=\int_{\Omega} f(\boldsymbol{\xi}) g(\boldsymbol{\xi}) W(\boldsymbol{\xi}) d \boldsymbol{\xi},
$$

with property $<\phi_{j}(\boldsymbol{\xi}), \phi_{k}(\boldsymbol{\xi})>=\delta_{j k}$, where $\delta_{j k}$ is the Kronecker $\delta$. Multivariate polynomials $\phi_{k}(\boldsymbol{\xi})$ are constructed as products of univariate polynomials only depending on a single random variable with sum of the orders of the univariate polynomials (total degree) at most $P$. The number of polynomials $K+1$ that is used in the PCE is determined as:

$$
K+1=\frac{(M+P) !}{M ! P !} .
$$

Galerkin projection of both sides of (2) on $\phi_{m}(\boldsymbol{\xi})$ finally leads to the following deterministic set of equations where the dependence on $\boldsymbol{\xi}$ has been eliminated

$$
\boldsymbol{V}_{m}=\sum_{m: \gamma_{k l m} \neq 0} \overline{\boldsymbol{Z}}_{k} \boldsymbol{I}_{l} \gamma_{k l m}
$$

with $\gamma_{k l m}=<\phi_{k}(\boldsymbol{\xi}) \phi_{l}(\boldsymbol{\xi}), \phi_{m}(\boldsymbol{\xi})>$ and where the summation is taken for all non-zero values of $\gamma_{\mathrm{klm}}$. Equation (6) represents a large deterministic system with $(K+1) N$ unknowns as in [5]. This system has $O\left(K^{2} N^{2}\right)$ memory complexity and $O\left(K^{3} N^{3}\right)$ computational complexity when using a direct solver. Memory complexity is reduced to $O\left(K N^{2}\right)$ and computational complexity to $O\left(n n z N^{2}\right)$ when using an iterative solver, where $n n z$ represents the number of non-zero values of $\gamma_{k l m}$. Numerical calculations show that for $P=1$, $n n z=O(K)$, while for $P>1, n n z=O\left(K^{1.5}\right)$ instead of $O\left(K^{3}\right)$ when all coefficients $\gamma_{k l m}$ would be different from zero.

Applying MLFMM can further decrease the computational complexity. In the FMM procedure the structure is divided into groups of sources and the matrix-vector product (MVP) is computed by calculation of the electric field radiated by one group of sources with group center $\rho_{s}$ at the group of observation points with group center $\rho_{o}$ [7]. This is done via the plane wave decomposition of the 2-D Green's function $G\left(\boldsymbol{\rho}(\boldsymbol{\xi}), \boldsymbol{\rho}^{\prime}(\boldsymbol{\xi})\right)$. One element of the matrix $\overline{\boldsymbol{Z}}_{k}$ of expansion coefficients is calculated as:

$$
\overline{\boldsymbol{Z}}_{i j, k}=\iint_{\Omega} \int_{s_{i}} G\left(\boldsymbol{\rho}(\boldsymbol{\xi}), \boldsymbol{\rho}^{\prime}(\boldsymbol{\xi})\right) d \boldsymbol{\rho}^{\prime} d \boldsymbol{\rho} \phi_{k}(\boldsymbol{\xi}) W(\boldsymbol{\xi}) d \boldsymbol{\xi},
$$

where $\boldsymbol{\rho}^{\prime} \in s_{j}$ and $\boldsymbol{\rho} \in s_{i}$, as testing and basis function are constant over segments $s_{i}$ and $s_{j}$. If the distance between the source group and observation group is sufficiently large, calculation of the interaction can be decomposed in an aggregation, translation and disaggregation step, i.e.

$$
\overline{\boldsymbol{Z}}_{k}=\int_{\Omega} \overline{\boldsymbol{D}}\left(\boldsymbol{\rho}(\boldsymbol{\xi}), \boldsymbol{\rho}_{o}\right) \overline{\boldsymbol{T}}_{q} \overline{\boldsymbol{A}}\left(\boldsymbol{\rho}^{\prime}(\boldsymbol{\xi}), \boldsymbol{\rho}_{s}\right) \phi_{k}(\boldsymbol{\xi}) W(\boldsymbol{\xi}) d \boldsymbol{\xi},
$$

where $\overline{\boldsymbol{T}}_{q}$ represents the diagonal translation matrix, where $\overline{\boldsymbol{A}}\left(\boldsymbol{\rho}^{\prime}(\boldsymbol{\xi}), \boldsymbol{\rho}_{s}\right)$ is the aggregation matrix of the source group and where $\overline{\boldsymbol{D}}\left(\boldsymbol{\rho}(\boldsymbol{\xi}), \boldsymbol{\rho}_{o}\right)$ is the disaggregation matrix of the observation group. Since we are dealing with independent random variables which describe the scatterer's geometry and source and observation group are separated, we assume that the aggregation matrix only depends on vector $\boldsymbol{\xi}_{s}$ which is a subset of vector $\boldsymbol{\xi}$ and is independent of subset $\boldsymbol{\xi}_{o}$ on which the disaggregation matrix depends. This means that the integral in (8) can be represented as a product of two integrals, one over subset $\boldsymbol{\xi}_{s}$ and one over subset $\boldsymbol{\xi}_{o}$. This leads to substantial simplifications as the PCE of disaggregation and aggregation matrix can now be determined separately. Furthermore, the translation matrix $\overline{\boldsymbol{T}}_{q}=\overline{\boldsymbol{T}}_{q}\left(\boldsymbol{\rho}_{s}, \boldsymbol{\rho}_{o}\right)$ depends on the group centers and also in case of stochastic variations we can keep these centers fixed. Hence, $\overline{\boldsymbol{T}}_{q}$ is a deterministic function, independent of $\boldsymbol{\xi}$, as long as the pertinent subsets $\boldsymbol{\xi}_{s}$ and $\boldsymbol{\xi}_{o}$ are disjunct.

In the MLFMM approach, groups are hierarchically divided into levels, where higher level groups consist of sets of lower level ones. To perform the MVP, first, in the aggregation step, the radiation pattern of the source group is sampled into outgoing plane waves (OPWs). On the lowest level this is done by multiplying the aggregation matrix of the group with the vector of current strengths of the sources. In the stochastic case, we need the PCE of the OPWs. The PCE coefficients for these OPWs, collected in vectors $\mathcal{O}_{m}^{\mathrm{PW}}$, are obtained via:

$$
\mathcal{O}_{m}^{\mathrm{PW}}=\sum_{m: \gamma_{k l m} \neq 0} \overline{\boldsymbol{A}}_{k} \boldsymbol{I}_{l} \gamma_{k l m}
$$

where matrices $\overline{\boldsymbol{A}}_{k}$ are the expansion coefficients of the aggregation matrix. OPWs of groups on higher levels are calculated via interpolation and shifting operations [7]. Via the translation step, OPWs are converted in incoming plane waves (IPWs) arriving at the observation group. IPWs at lower levels are calculated via anterpolation and shifting operations [7]. Finally, on the lowest level, stochastic IPWs are evaluated at the observations points in the disaggregation step. This leads to the coefficients $\boldsymbol{V}_{m}$ in (6), given by:

$$
\boldsymbol{V}_{m}=\sum_{m: \gamma_{k l m} \neq 0} \overline{\boldsymbol{D}}_{k} \mathcal{I}_{l}^{\mathrm{PW}} \gamma_{k l m}
$$

where matrices $\overline{\boldsymbol{D}}_{k}$ are the expansion coefficients of the disaggregation matrix and $\mathcal{I}_{l}^{\mathrm{PW}}$ represents the expansion coefficients of the IPWs. In the MLFMM approach advocated here, (9) and (10) are calculated at the lowest level of the MLFMM tree. The cost of these steps in a deterministic MLFMM scheme is $O(N)$ [7]. From (9) and (10), it can be seen that the complexity is now increased to $O(n n z N)$. But, since the aggregation and disaggregation matrices only depend on subsets of $\boldsymbol{\xi}$, many expansion coefficients $\overline{\boldsymbol{A}}_{k}$ and $\overline{\boldsymbol{D}}_{k}$ are zero, and the complexity is substantially decreased, as will be numerically demonstrated in the next section. The complexity of other operations (interpolation, shifting, translation, anterpolation) is $(K+1)$ times higher than in the deterministic case, since OPWs and IPWs are described via PCE. For dense structures, complexity of these operations would be $O(K N)$. In a similar way, the matrices $\bar{Z}_{k}$ are however sparse, and the complexity of MVPs in the MoM can be reduced, as will be demonstrated in the next section. 


\section{NuMERICAL EXAMPLE:}

\section{A. Scattering by a finite periodic $2 D$ array of PEC strips}

We consider TM-scattering by a periodic but finite 2D array of PEC strips with varying widths $w$ as depicted in Fig. 1 for the four strips case. The $x$-coordinates of the position vectors of the centers of the strips remain fixed with constant spacing $T$. On the other hand, the $y$-coordinates vary as described by relative heights $h$ w.r.t. the nominal $y$-values. These nominal values are again equally separated by a spacing $T$. The variability of the structure is hence described by vectors of widths $w$ and relative heights $h$ which are chosen to be independent uniformly distributed random variables. The widths of the strips vary between $0.5 T-\lambda / 20$ and $0.5 T+\lambda / 20$, with $T=\lambda$, and the heights of the strips between $-\lambda / 20$ and $\lambda / 20$, where $\lambda$ is the wavelength of the incident wave, i.e. we introduce $20 \%$ variations compared to nominal width $w=0.5 T$. The incident wave is a TMpolarized plane wave impinging under an angle $\alpha=3 \pi / 4$ with the positive $x$-axis and frequency $f=2.45 \mathrm{GHz}$.

In the MLFMM scheme, the structure is divided in square boxes with fixed side $T$ and centers indicated by $B_{0}, B_{1}, B_{2}$ and $B_{3}$ which coincide with the nominal position vectors of the centers of the strips. In order to demonstrate the benefits of using the MLFMM, the number of strips will be increased to 4 by 4,8 by 8 and 16 by 16, keeping the nominal center positions on a regular $T$ by $T$ grid. At the lowest MLFMM level the box size also remains $T$ by $T$.

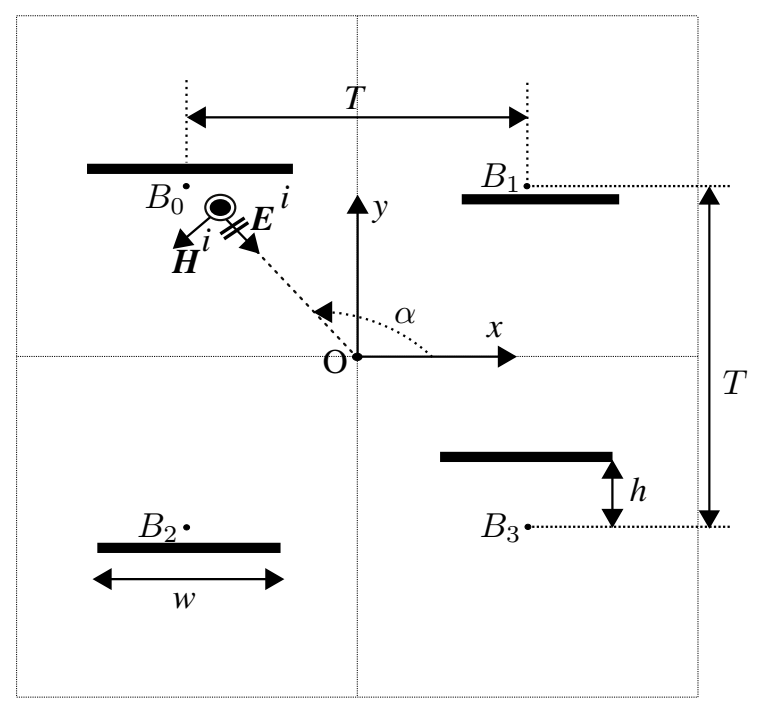

Fig. 1. Periodic 2D array of PEC strips. Widths $w$ and heights $h$ are random. For clarity, here, the case with only four strips is shown.

The PCE uses multivariate orthonormal Legendre polynomials which are the proper functions to model uncertainties in the case of uniform distributions. In the MoM approach, matrix elements that describe interactions on the same strip depend on a single random variable, i.e. the width, and matrix elements that describe interactions between two strips depend on four random variables, i.e. two widths and two heights. Hence, in this example, the $M$ dimensional integral in (7) is

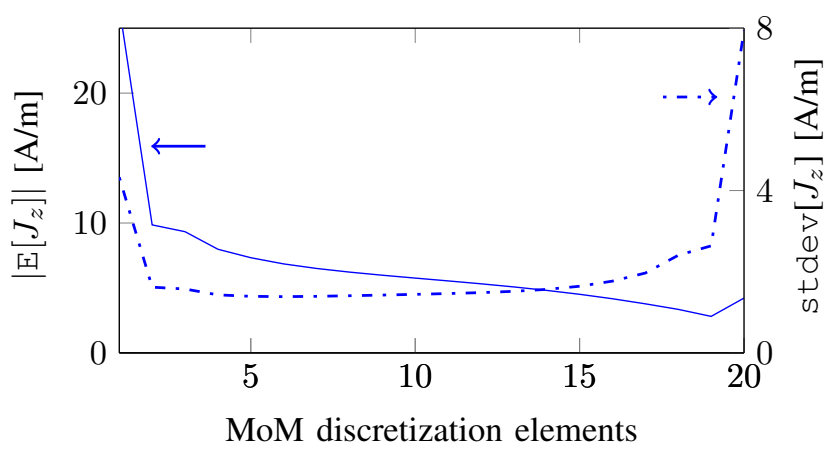

Fig. 2. Magnitude of the mean value (full line) and standard deviation (dash-dotted line), of the current distribution on the top left strip for the $4 \times 4$ example and total degree $P=2$.

reduced to either a one dimensional or a four dimensional integral. When calculating $\overline{\boldsymbol{A}}_{k}$ and $\overline{\boldsymbol{D}}_{k}$, matrix elements only depend on two random variables.

The current on each of the strips is modeled using 20 equal length subdivisions with piecewise constant basis functions. The total number of unknowns in the MoM grows from $N=80$ in the $2 \times 2$ case to $N=5120$ in the $16 \times 16$ case. The total number of random variables also grows with a factor 4 from $M=8$ to $M=512$. For this example, the quantities of interest (surface current, RHS, impedance matrix elements) are modeled using expansions with highest polynomial order, i.e. total degree, $P=2$. Our experience shows that $P=2$ suffices to describe the wanted statistics with acceptable accuracy. In the last example with 512 random variables, the total number of polynomials in (5) is so large, i.e. 131841, that we have restricted ourselves to the $P=1$ case.

Fig. 2 represents the mean value and the standard deviation of the induced current for top left strip of the scatterer for the $4 \times 4$ case. Results from straightforward application of the MoM-SGM combination and from MoM-SGM with MLFMM acceleration are indistinguishable on the scale of the figure. The most important numerical difference between the two approaches is due to the fact that the MoM-SGM approach uses a PCE for $\overline{\boldsymbol{Z}}(\boldsymbol{\xi}), \boldsymbol{I}(\boldsymbol{\xi})$ and $\boldsymbol{V}(\boldsymbol{\xi})$ in (1), while the MoMSGM-MLFMM does the same for $\boldsymbol{V}(\boldsymbol{\xi})$ and $\boldsymbol{I}(\boldsymbol{\xi})$ but on top of that introduces separate PCEs for the disaggregation and aggregation matrices $\bar{D}(\boldsymbol{\xi})$ and $\overline{\boldsymbol{A}}(\boldsymbol{\xi})$ in (8), possibly leading to additional truncation errors in (9) and (10). This truncation error when using SGM is discussed in [8], showing that this error decreases for increasing polynomial order $P$ and for decreasing variations of the random variables. Another numerical difference arises when determining the radiated field due to a group of sources in an MLFMM box. In the MoMSGM approach this field is directly obtained using the Green's function and the PCE of the currents. In the MoM-SGMMLFMM approach this field emerges from the group's OPW expansion and depends both on the PCE of these OPWs and on the number of sampling directions. In order to illustrate the numerical differences, results for the mean and the variance of the magnitude of the center current on subdivision 10 of the top left strip for the $4 \times 4$ and $8 \times 8$ examples are shown in Table $\mathrm{I}$, for $P=1$ and $P=2$. 
TABLE I

MEAN AND VARIANCE OF THE MAGNITUDE OF THE CENTER CURRENT OF THE TOP LEFT STRIP FOR DIFFERENT METHODS

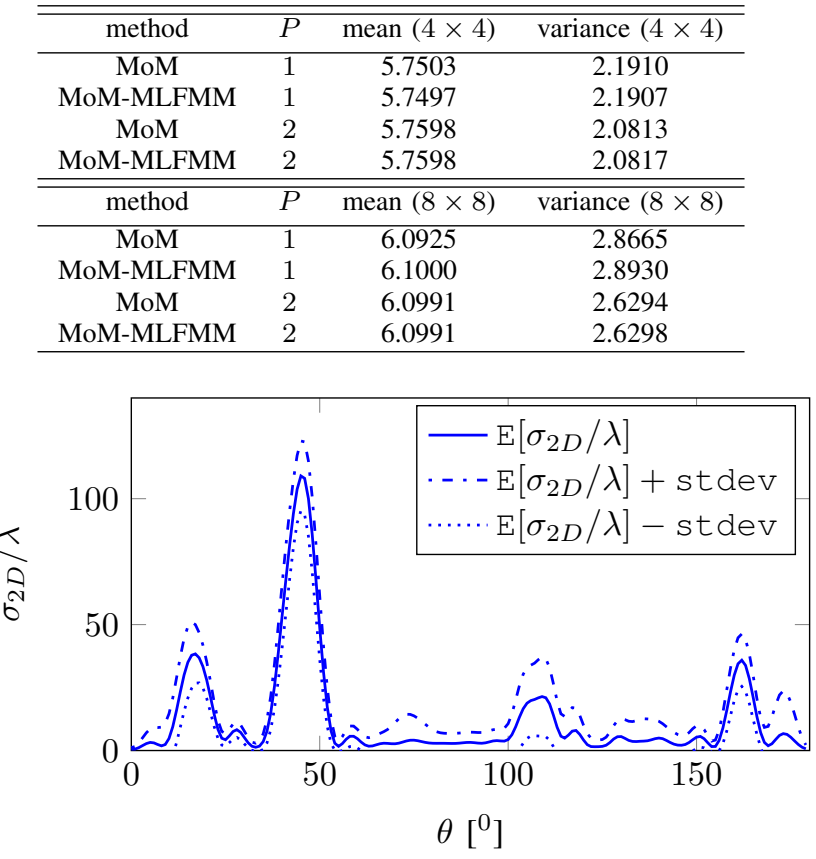

Fig. 3. Mean RCS p.u.1. with \pm one standard deviation variation for structure with $8 \times 8$ strips for polynomial order $P=2$.

The radar cross-section (RCS) per unit length (p.u.l.), also called scattering width, is given by

$$
\sigma_{2 D}=\lim _{\rho \rightarrow \infty} 2 \pi \rho \frac{\left|E_{z}^{s}\right|^{2}}{\left|E_{z}^{i}\right|^{2}},
$$

where $\rho$ is the distance to the origin $O$ in Fig. $1, E_{z}^{i}$ is the incident electric field and $E_{z}^{s}$ is the scattered electric field which can be derived from the induced currents or in the MLFMM approach, by using the OPWs expansion at the highest level. Fig. 3 shows the mean value and variations around this mean value with maximum variability of one standard deviation for the structure with $8 \times 8$ strips. It can be seen that the RCS p.u.l. is the highest in the specular reflection direction and that the radiation pattern shows a number of side lobes. The \pm one standard deviation of these lobes is quite substantial.

Finally, in Table II, in order to demonstrate the computational efficiency of the proposed MLFMM approach, we present the $\mathrm{CPU}$ time for one matrix-vector product (MVP) for different cases and polynomial orders, again comparing the straightforward MoM-SGM approach to the MLFMM accelerated one. In this table, $N$ is the number of unknowns in the MoM (320: $4 \times 4$ case; 1280: $8 \times 8$ case; 5120: $16 \times 16$ case), $P$ is the maximum degree of the multivariate polynomials, $K+1$ is the corresponding total number of polynomials corresponding to a particular $P$-value and $n n z$ is the number of non-zero values of $\gamma_{k l m}$ in (6). Remark that the case $P=0$ simply corresponds to using the nominal value of all variables, i.e. the simple deterministic case. Further remark that $n n z$ has to be weighed against the maximum of $K^{3}$ when all $\gamma_{k l m}$ would be different from zero. Table II shows that the acceleration due to the MLFMM is very substantial for the largest $(16 \times 16)$ scatterer. The crossover point is found at about $N=1000$, as is also the case for traditional (deterministic) MLFMM.

TABLE II

CPU TIME FOR ONE MVP PRODUCT

\begin{tabular}{cccccc}
\hline \hline$N$ & $P$ & $K+1$ & $n n z$ & MLFMM [s] & MoM [s] \\
\hline 320 & 0 & 1 & 1 & 0.0017 & 0.0012 \\
320 & 1 & 33 & 97 & 0.0658 & 0.04822 \\
320 & 2 & 561 & 37521 & 1.8509 & 1.5181 \\
1280 & 0 & 1 & 1 & 0.0103 & 0.0184 \\
1280 & 1 & 129 & 385 & 1.334 & 2.3681 \\
1280 & 2 & 8385 & 2171457 & 118.99 & 204.33 \\
5120 & 0 & 1 & 1 & 0.0518 & 0.2877 \\
5120 & 1 & 513 & 1537 & 25.34 & 149.07 \\
\hline
\end{tabular}

\section{CONCLUSION}

In this letter, two-dimensional TM-scattering at an electrically large, stochastically varying, PEC scatterer was used to demonstrate how the statistics of induced currents and of the radiation pattern can be found by optimally combining the MoM, the SGM and the acceleration due to the MLFMM. The SGM is an intrusive method allowing to replace the original stochastic problem by an equivalent, but much larger, deterministic problem. As the PEC strip array example in the paper demonstrates, the solution of this larger deterministic problem, greatly benefits from the MLFMM by taking into account the particularities of the matrix-vector product which arises from the application of the SGM.

Further research is needed to include dielectrics objects to extend this approach to the 2D analysis of multiconductor transmission lines, and also to the $3 \mathrm{D}$ case in order to find out if all conclusions remain valid.

\section{REFERENCES}

[1] D. B. Xiu and G. E. Karniadakis, "The Wiener-Askey polynomial chaos for stochastic differential equations," SIAM Journal on Scientific Computing, vol. 24, no. 2, pp. 619-644, 2002.

[2] D. Xiu, "Efficient collocational approach for parametric uncertainty analysis," Communications in Computational Physics, vol. 2, no. 2, pp. 293-309, Apr. 2007.

[3] R. G. Ghanem and P. D. Spanos, Stochastic Finite Elements. A Spectral Approach. New York:Springer-Verlag, 1991.

[4] D. Vande Ginste, D. De Zutter, D. Deschrijver, T. Dhaene, P. Manfredi, and F. Canavero, "Stochastic modeling-based variability analysis of onchip interconnects," IEEE Transactions on Components, Packaging and Manufacturing Technology, vol. 2, no. 7, pp. 1182-1192, Jul. 2012.

[5] T. El-Moselhy and L. Daniel, "Variation-aware stochastic extraction with large parameter dimensionality: Review and comparison of state of the art intrusive and non-intrusive technique," in 2011 12th International Symposium on Quality Electronic Design (ISQED 2011), 14-16 March 2011, Santa Clara, CA, USA, 2011, pp. 508-517.

[6] C. Chauvière, J. S. Hesthaven, and L. C. Wilcox, "Efficient computation of RCS from scatterers of uncertain shapes," IEEE Transactions on Antennas and Propagation, vol. 55, no. 5, pp. 1437-1448, May 2007.

[7] W. C. Chew, J. M. Jin, E. Michielssen, and J. Song, Fast and Efficient Algorithms in Computational Electromagnetics. Norwood, MA: Artech House, 2001.

[8] B. J. Debusschere, H. N. Najm, P. P. Pébay, O. M. Knio, R. G. Ghanem, and O. P. L. Maitre, "Numerical challenges in the use of polynomial chaos representations for stochastic processes," SIAM J. Sci. Comput., vol. 26, no. 2, pp. 698-719, Feb. 2005. 